\title{
Смысловые характеристики терминов цифровой экономики как фактор эффективного делового общения (на примере английского языка)
}

\author{
Глушкова Н.М., Харченко М.Г. \\ Финансовый университет при Правительстве Российской Федерации (Финансовый университет), \\ Россия, 125993 (ГСП-3), г. Москва, Ленинградский проспект, 49 \\ E-mail: NMGlushkova@fa.ru,margrih@mail.ru
}

\begin{abstract}
Аннотация. Дано системное описание ряда актуальных на данный момент цифровых терминов, обладающих множеством семантических характеристик, правильное понимание которых является одним из важных факторов эффективного делового общения. Используя примеры цифровых терминов на английском и русском языках в ходе сопоставительного анализа, авторы показывают зависимость семантических характеристик цифровых терминов от таких факторов, как морфологические и стилистические особенности, синонимия, дифференциация по сферам общения и возможностям употребления для различных ситуаций. Описанные в статье примеры цифровой терминологии обновляют материал по исследуемой теме и мотивируют читателя к дальнейшему поиску информации по данной теме для дальнейшей научной работы и самообучения в аспекте глобальной цифровизации.
\end{abstract}

Ключевые слова: цифровая терминология, семантические характеристики, термин, цифровизация.

Для цитирования: Глушкова Н. М., Харченко М. Г. 2020. Смысловые характеристики терминов цифровой экономики как фактор эффективного делового общения (на примере английского языка). Вопросы журналистики, педагогики, языкознания, 39 (2): 280-288. DOI 10.18413/2712-74512020-39-2-280-288

\section{Semantic characteristics of digital economy terms as a factor of effective business communication (using the example of english)}

\author{
Nadezhda M. Glushkova, Marina G. Kharchenko \\ Financial University under the Government of the Russian Federation (Financial University), \\ 49 Leningradsky Prospekt, Moscow, 125993, GSP-3, Russia \\ E-mail: NMGlushkova@fa.ru,margrih@mail.ru
}

\begin{abstract}
A systematic description of a range of digital terms with a large number of semantic characteristics is given, the correct understanding of which is one of the important factors contributing to effective business communication. Based on the opinion of some researchers of digital terminology, the authors of the article use a comparative approach to the study of digital terms in English and Russian to identify the dependence of semantic characteristics of digital terms on such factors as morphological and stylistic features, synonymy, differentiation by spheres of communication and the possibility of use in different situations. The authors claim that an incorrect understanding of semantic characteristics, on the one hand, and an insufficiently detailed scientific study of digital terms and their lack of systematization, on the other hand, can negatively affect the effectiveness of business communication. The results of the scientific review contribute to the understanding of the semantic characteristics of digital terms and their systematization, update the material on the topic under study and motivate the reader to further search for information to expand scientific study and self-learning in the aspect of global digitalization.
\end{abstract}


Keywords: digital terminology, term, semantic characteristics, digitalization.

For citation: Glushkova N.M., Kharchenko M.G. 2020. Semantic characteristics of digital economy terms as a factor of effective business communication (using the example of English). Issues in Journalism, Education, Linguistics, 39 (2): 280-288 (in Russian). DOI 10.18413/2712-7451-2020-39-2-280-288

\section{Введение}

Язык как социальное явление составляет неотъемлемую часть всех общественных отношений. В эпоху развития цифровой экономики изменения, происходящие в языке, приобретают особый смысл. Цифровизация постепенно охватывает такие сферы жизни и деятельности людей, как пассажирские и грузовые перевозки, туризм, общественное питание, розничная торговля, госуправление, промышленность, медицина и другие.

Слова как составляющие лексики языка испытывают на себе воздействие экстралингвистических факторов, влияющих на развитие языка. Такие вопросы, как значения новых слов и выражений, связанных с цифровой экономикой, их синонимия, дифференциация лексики по сферам общения представляют интерес для современной лингвистики. Для большинства людей, общающихся в условиях цифровой экономики, смысл и история цифровой терминологии, ее стилистические свойства и возможности употребления в различных ситуациях неизвестны или не до конца ясны.

Логично предположить, что информационное пространство оперативно реагирует на потребность общества понять процессы, происходящие в экономике, обществе и языке. C. Рагимова [2019], одна из авторов онлайн-версии журнала Forbes, освещает необходимый минимум лексики цифровой экономики, которым должны владеть все, кто с ней соприкасается. В частности, приводятся примеры программных систем, задействованных в организации бизнеса: BPM (Business Process Management), HRM (Human Resources Management), CRM (Customer Relationship Management), SAP (Resume Matching) и многие другие [Что такое цифровая экономика..., 2019; Рагимова, 2019].

Современная система образования, которая призвана отвечать на запросы глобального рынка труда и повышать востребованность выпускников, нуждается в соответствующих действиях, информационных источниках и учебных изданиях, которые познакомят широкую аудиторию с цифровизацией мира. Министерство цифрового развития, связи и массовых коммуникаций Российской Федерации совместно с Аналитическим центром при Правительстве Российской Федерации организовали круглый стол «Глоссарий цифровой экономики», на котором была высказана точка зрения, что базовая терминология цифровой экономики, принятая в мировых производствах Китая, США и Европы, все еще не полностью понятна многим пользователям. В России нужно выработать единые понятия и термины цифровой экономики, определить их характеристики, принять меры по синхронизации существующих терминов, чтобы облегчить работу участникам цифрового рынка и способствовать формированию культуры потребления цифровых услуг. По мнению участников круглого стола, эти проблемы могут быть решены, если выработать единую терминологическую базу и создать глоссарий цифровой экономики [Румянцев и др., 2018].

Понимание различий смысловых характеристик цифровых терминов лежит в основе эффективного делового общения, в связи с чем возникла необходимость для специалистов и всех тех, кто вынужден общаться в условиях цифровой экономики, комплексно изучать характеристики ее терминов с учётом социокультурных особенностей коммуникации в русскоязычной и англоязычной лингвокультурах. Несмотря на то, что с расширением деловых контактов цифровая терминология все активнее проникает в коммуникативное пространство современного общества, количество работ, посвященных ее лингвистическому анализу и влиянию экстралингвистических факторов, пока недостаточно. 
В данной статье мы приводим ряд примеров интерпретации и описания англоязычных и русскоязычных цифровых терминов.

\section{1. Масштабность использования английского языка и его включенность в русскоязычную действительность}

Новые тенденции и идеи в экономике и менеджменте, связанные с цифровизацией, привели к обновлению языка для коммуникации, изменению ряда грамматических форм и стилей общения. Наиболее ярко это проявляется в Интернете. Глобальное распространение английского, его взаимодействие с другими языками и дальнейшая цифровизация привели к появлению новых терминов, смысловые характеристики которых отличаются от прежней терминологической системы. Иными словами, язык общения претерпевает изменения под воздействием как лингвистических, так и экстралингвистических факторов, которые напрямую связаны с переходными моментами в жизни языкового сообщества.

В частности, можно показать, как некоторые слова и термины расширяют свои значения, чтобы соответствовать новым ситуациям, на следующих примерах:

- in-memory - технологии управления данными, анализа их в оперативной памяти [Иванов, 2018].

Пример контекста:

In computer science, in-memory processing is an emerging technology for processing of data stored in an in-memory database [In-memory processing].

В данном случае мы наблюдаем характерную для языка тенденцию к упрощению языкового материала и закреплению тех его форм, которые наиболее емко и доступно передают смысл как носителям языка, так и изучающим его. Даже не зная точного перевода, собеседник по контексту и форме слова, прикрепленному префиксу, может ориентироваться в ситуации.

- Big Data - огромные объемы информации, собранной из самых разных источников, потенциально представляющие ценность [Mozyrko, 2015].

Например:

Every field has its own terminology and thus, there are a number of Big Data terms to know while starting a career in Big Data [Verma, 2018].

Данный пример иллюстрирует, как язык обретает новый термин, сочетая простую в обращении и запоминании лексику, придавая ему статус более удобного, легкого в употреблении термина, короткого по объему и разнообразного в значении.

- Cloud Computing - метод, который дает возможность бизнесу и частным пользователям запускать приложения, хранить данные и пользоваться сервисами без необходимости покупать собственные сервера, системы хранения и прочее оборудование [Рагимова, 2019].

Например:

In the simplest terms, cloud computing means storing and accessing data and programs over the Internet instead of your computer's hard drive [Griffith, 2016].

Как видно из контекста, язык сочетает тенденцию к упрощению с присущей ему образностью (Cloud - англ. облако). Полагаем, что отчасти эта черта - также следствие особенностей человеческого мышления, яркий образ способен лучше запечатлеться в памяти, вызвать стойкие ассоциации.

- Lyft - услуги по перевозке пассажиров (такси) [ТАКСИМО. Официальный сайт «Lyft» такси в Москве].

Например:

Lyft is a very convenient service when you need a ride [Lift Delivery, 2019]. 
Пример данного слова иллюстрирует синергию языка, цифровых технологий и экономики. Популярный бренд такси закрепился в обиходе для обозначения услуг пользования не только машин, но и велосипедов, и электросамокатов. Сам термин основан на видоизмененном слове "lift", который в ряде устойчивых выражений устойчиво ассоциируется с транспортом (togivealift - подвезти)

- SoLoMo (Social+Local+Mobile) - мобильные технологии, которые помогают взаимодействовать с потребителями через экраны мобильных устройств с учетом геолокации и с использованием социальных медиа [Новый маркетинг, 2012].

Например:

SoLoMo is an acronym for Social+Local+Mobile marketing and it is an increasingly common term referring to a new marketing approach [Ruiz, 2015].

Рассматриваемый термин является прямым примером взаимодействия маркетинга и цифровых технологий, когда из ряда прилагательных появляется универсальное слово, включающее в себя емкое информационное поле, которое до появления термина требовало более пространного и развернутого описания.

Как показывают примеры, функциональная дифференциация английского языка и его многообразие возникают как результат экстралингвистического воздействия.

\section{2. Термины цифровой экономики как объект англо-русских переводческих сопоставлений}

Вначале необходимо отдельно отметить ключевые факторы отбора терминов цифровой экономики для сопоставления. Мы хотим представить обзор ряда определений, отвечающих за взаимозависимый процесс слияния понятий информации и экономики. Без своевременной информации невозможно успешное развитие и рост экономики. Вместе с тем именно экономическая успешность и мотивированность является фундаментом для целого ряда информационных исследований. Дополнительный критерий выбора того или иного термина для анализа заключается в его актуальности и одновременно относительной новизне в русском языке.

Руководствуясь правилом освещения понятий синергии «информация - бизнес» от целого к частному, прежде всего необходимо получить представление о направлении "Business Intelligence" (BI). В мировой экономике оно существует несколько десятилетий и активно используется для описания, анализа и прогнозирования экономических событий. В современном экономическом обиходе мы можем встретить разнообразные варианты перевода в зависимости от понятия, которое подразумевают авторы работы. Приводится как вариант «интеллектуальный анализ данных» [Корнеев и др., 2000], так и калькированный вариант «бизнес-интеллект» [Артемьев, 2003]. В зависимости от контекста, это понятие может передавать процесс сбора многоаспектной информации либо трансформацию данных в знания, азнаний в действия для компаний, желающих получить прибыль. В этом случае возникаетвариант термина «бизнес-аналитика» [Паклин, Орешков, 2013]. Разграничивая понятия аналитики и ее более узкого варианта Business Intelligence, отметим, что основная задача ВI заключается в формулировании действий и решений для предпринимателя [Что такое Business Intelligence, 2015].

Еще один взгляд заключается в понимании бизнес-интеллекта как наборе инструментов, программном обеспечении для оперирования большим количеством данных [Артемьев, 2003]. За основу мы возьмем достаточно полное определение словаря «Академик»: «классификация и анализ данных, собираемых при ведении бизнеса, чтобы на их основе принимать более эффективные бизнес-решения и добиваться конкурентных преимуществ» [Business intelligence,2019]. Примечательно, что этот словарь отражает неоднозначность понятия и дает три варианта переводов: «бизнес-аналитика», «система анализа 
деловых данных», «средства бизнес-аналитики». Англоязычное понятие также соединяет в себе процесс, инструменты и конечный результат - набор решений:

"Business Intelligence is a process of collecting, integrating, analyzing and presenting the data for a better understanding of decision-making. This process is carried out through software services and tools" [Business Intelligence vs Data Science - 4 Ways to Tell Them Apart, 2019].

Зарубежные авторы часто сопоставляют и равно высоко оценивают актуальность терминов "Business Intelligence" и "Data Science" (наука о данных):

"Business Intelligence and Data Science are two of the most recurring terms in the digital era" [Business Intelligence..., 2019].

При этом подчеркивается, что сфера науки о данных шире бизнес-аналитики: несмотря на объединяющий их объект исследования (сложные данные, BigData), наука о данных оперирует сложными статистическими алгоритмами для прогнозирования будущего, в том числе бизнес-сферы. Бизнес-аналитика, в свою очередь, концентрируется на узких, актуальных экономических вопросах:

"BI is about developing dashboards, creating business insights, organizing data and extracting information that would help the businesses to grow. ... Data Science is much more complex compared with Business Intelligence" [Business Intelligence..., 2019].

Следующий термин представляет более узкое понятие "DataEngineering" или «инжиниринг данных», а также соседствующие с ним термины "Data Analysis" (анализ данных). Эти сферы наступившего будущего, помимо информации о развитии событий и явлений, или предиктивного моделирования, предлагают целый набор возможностей для экономики общества. Более того, на данный момент профессия дата-инженера или датасаентиста считается одной из самых востребованных. Однако мы не случайно рассматриваем информационно-технические профессии в рамках экономической применимости. Алгоритмы и данные важны не сами по себе, а в контексте протекающих в обществе бизнес-процессов. Деятельность любого аналитика в первую очередь нацелена на повышение прибыли компании. Соответственно, успешный представитель этой профессии должен обладать знаниями об экономических моделях, структуре и процессах на предприятии.

Для понимания этого феномена и его роли в цифровой экономике разберем обработку больших данных (Bigdata) с максимально общей точки зрения. В структуре работы с данными выделяют равноценные по вкладу в процесс термины-профессии:

1) Дата-аналитики (The Data Analysts) при помощи языков программирования, электронных таблиц и инструментов бизнес-аналитикиописывают и подразделяют на категории существующие и актуальные информационные данные.

2) Дата-саентисты (The Data Scientists) задействуют алгоритмы для создания прогнозов о будущем развитии событий в отношении имеющихся данных и на их основе.

3) Дата-инженеры (The Data Engineers) - специалисты, которые с помощью языков программирования обеспечивают процесс достоверной информацией, отвечают за постоянный доступ к ней в базах данных [WhatisDataEngineering? 2018].

4) Дата-инжиниринг (или второй вариант: инженерия данных) связан с данными, их получением, расположением и сохранением в базах данных, а в дальнейшем - обработкой и перенаправлением для анализа и прогнозирования. Основная задача инженеров состоит в создании надежной инфраструктуры для больших объемов данных [Кто такие..., 2019].

Если рассмотреть данные термины с лингвистической точки зрения, то можно понять, что у этих профессий существует общее происхождение: они возникли как стремительно набирающие популярность жаргонизмы, которые закрепились в языке. Одинаково по звучанию и по написанию термины представляют собой характерное для современности заимствование иностранного слова с сохранением его приблизительного произношения, несмотря на чужеродность формы. Причина такой словообразовательной и переводческой тенденции, свидетельствующей о скорости внедрения слова в язык, кроется в высокой востребованности этих знаний и навыков. Мы также наблюдаем различие в пере- 
водческих методиках, использованных при переводе на русский язык трех профессий, указанных выше. В случае наличия в языке аналога профессии с максимально обобщённымзначением и большими возможностями для употребления вторая часть термина передается русским аналогом (дата-инженер, дата-аналитик), однако в случае с "data scientist" тенденции перевода не так однозначны. Во-первых, мы встречаем в сети разные варианты их транскрипционной передачи («дата-саентисты» и «дата-сайентисты») [Новый маркетинг, 2012]. Во-вторых, в силу того, что с 2010-х годов наука о данных (“datascience”) выделена в образовательных стандартах как практическая межотраслевая сфера деятельности, академическая дисциплина и раздел информатики, существует менее популярный в профессиональном обращении, но более академически оформленный вариант «ученый по данным» [Дата-сайентисты..., 2018]. Множество русских эквивалентов также пополняют варианты «дата-ученый», «дата-специалист» [Дата-учёный..., 2016] и наиболее описательный перевод из встречаемых - «специалист по изучению данных» [Специалист..., 2019].

Разнообразие вариантов перевода имеет под собой логичное социальноэкономическое обоснование: профессии находятся на этапе становления, равно как и актуальные образовательные программы.

Далее рассмотрим явление, несущее огромные возможности именно для сферы экономики и производства. Появление термина «Интернет вещей» (“Internet of Things” or "ІоТ”) в первую очередь связано с целью интегрировать и развить общественные и экономические процессы в той мере, чтобы минимизировать необходимость человеческого участия в максимально возможном количестве операций и тем самым повысить эффективность, точность и экономическую прибыльность любого предприятия.

Приведем несколько определений данного понятия для формирования более четкого представления о нем:

The Internet of Things, or IoT, refers to the billions of physical devices around the world that are now connected to the internet, all collecting and sharing data. Connecting up all these different objects and adding sensors to them adds a level of digital intelligence to devices [Internet of things, 2018].

В этом наиболее простом из определений акцент ставится на усовершенствовании привычных предметов и процессов с доступом ко всемирной сети и сенсорным технологиям, позволяющим собирать и обмениваться информацией без участия человека. К понятию Интернета вещей относят, например, возможность выключать электричество дома из телефонного приложения или беспилотное движение автомобиля.

The IoT is a giant network of connected things and people - all of which collect and share data about the way they are used and about the environment around them. ... Devices and objects with built in sensors are connected to an Internet of Things platform [Clark, 2016].

Еще один аспект, который необходимо учитывать при изучении Интернета вещей это существование единой сетевой платформы, где интегрируется вся информация, полученная от встроенных сенсоров. Далее к этим данным подключается анализ данных. Мощные платформы отбирают полезную информацию и отсеивают ненужную. В дальнейшем данные, которые сочли нужными, используют в совершенствовании образцов поведения, создании новых алгоритмов и устранении операционных ошибок, в том числе до их появления.

The internet of things, or IoT, is a system of interrelated computing devices, mechanical and digital machines, objects, animals or people that are provided with unique identifiers (UIDs) and the ability to transfer data over a network without requiring human-to-human or human-tocomputer interaction (Internet of things (IoT) [Internet..., 2020].

Данное определение, во-первых, значительно расширяет сферы использования Интернета вещей, т.к. он затрагивает уже одушевленные существа. В частности, приводятся примеры ферм с животными со вживленными микрочипами (biochip transponder) или человека, в сердце которого находится имплант, отслеживающий все происходящие процес- 
сы (a heart monitor implant). Во-вторых, мониторинг, сбор информации, ее анализ и получение результатов не требуют не только взаимодействия уровня «человек - человек», но и «человек - компьютер». Системы наделяются почти полной автономностью процесса, хотя человек может взаимодействовать с устройствами, чтобы настроить их, дать инструкции или получить доступ к информации.

Вследующем фрагменте отметим, как Интернет вещей применим в экономике: "The use of smart devices will also likely mean a competitive advantage for businesses that use them strategically. For instance, by tracking data about energy use and inventory levels, a firm can significantly reduce its overall costs" [Kenton, 2020].

В данном примере знание об Интернете вещей выступает как стратегическое экономическое преимущество, способное как сокращать расходы предприятия, так и выполнять, казалось бы, более творческую часть предпринимательства - совершенствовать маркетинговые ходы и тем самым повышать продажи.

С лингвистической точки зрения интересно отметить, что новое понятие уже имеет своеобразное продолжение и развитие в ряде терминов, которые ответвляются от более общего понятия. Постепенно выделилась более узкая область, относящаяся к энергии (Internet of Energy (IoE)), которая направлена на автоматизацию и совершенствование энергетической инфраструктуры и производства: “The term is derived from the increasingly prominent market for Internet of Things (IoT) technology, which has helped develop the distributed energy systems that make up the IoE" [Chen, 2020]. Следующим родственным термином "Internet of Things" стало его производное - "The Internet of Everything" (Интернет всего или Интернет-всего) - одно из самых перспективных направлений деятельности [Ваннах,2013]. В последнем термине собрано множество составляющих современного общества (люди, действия, предметы и информация), которые можно объединить в одну глобальную сеть: "IoE is the intelligent connection of people, process, data and things. The Internet of Everything (IoE) describes a world where billions of objects have sensors to detect measure and assess their status; all connected over public or private networks using standard and proprietary protocols" [Banafa, 2016].

\section{Заключение}

Мы коснулись только некоторых аспектов анализа цифровой терминологии:

1. Проведено сопоставление актуальных и наиболее часто используемых цифровых терминов из различных экономических и социальных сфер, в результате чего очевидно, что для изучения цифровой терминологии первоначальным является лексикология, где смысловые характеристики терминов занимают особое место, обеспечивая их правильное использование.

2. Приведенные примеры использования цифровых терминов обнаруживают определенную тенденцию к сближению между языками благодаря социолингвистическим процессам, особенно в части их словообразовательных возможностей.

2. Рассмотренные примеры цифровых терминов подтверждают, что активное развитие цифровой экономики способствует быстрому расширению и обновлению языка сообщества, в котором понимание появляющихся лексических единиц можно получать с помощью таких переводческих методов, как транскрипция, транслитерация, калькирование и описательный перевод.

3. Указанные в статье термины показывают, что частота и точность употребления новых цифровых понятий в языке сообщества зависит от понимания коннотаций конкретного слова, его характерных словосочетаний, сопутствующего контекста и его положительного или отрицательного значения в зависимости от ситуации применения.

4. Такие вопросы, как перенос англоязычных понятий в русскую экономическую сферу, имеет ряд особенностей, которые необходимо учитывать при переводе, трактовке и 
употреблении терминов для делового общения. Это требует дальнейшего лингвистического анализа, систематизации и описания семантических характеристик актуальных терминов.

Результаты работы могут быть рассмотрены в качестве мотивации обучающих учреждений включать современные термины цифровой экономики в учебные курсы высшей и средней школ с целью лучшей подготовки будущих специалистов к участию в деловой коммуникации. Задача лингвистов в дальнейшем видится в подробном и оперативном изучении новых заимствований и анализа их с точки зрения семантической структуры, семиотической денотации, метасемиотики, коннотации, а также эволюции заимствованного значения каждой лексической единицы. Все это ведет общество к созданию единого языка общения, понятного как в деловой сфере, так и в обычной жизни, особенно в условиях цифровой экономики.

\section{Список источников}

1. Ваннах M. 2013. Через «Интернет вещей»к «Интернету всего». Компьютерра. URL: https:/www.computerra.ru/183215/cherez-internet-veshhey-k-internetu-vsego/ (дата обращения: 16.02.20)

2. Дата-сайентисты - редкая порода профессионалов. 2018. URL: http://rtbinsight.ru/articles /data-sajentisty-redkaya-poroda-professionalov.html (датаобращения: 16.02.20)

3. Дата-учёный I и II типа. 2016. URL: http://datascientist.one/data-scientist-types/ (дата обращения: 16.02.20)

4. Иванов Н. 2018. Технология In-memoryComputing: как применять и зачем? URL: https://www.crn.ru/news/detail.php?ID=124497 (дата обращения: 16.02.20)

5. Корнеев В.В., Гареев А.Ф., Васютин С.В., Райх В.В. 2000. Базы данных. Интеллектуальная обработка информации. М., Нолидж, 352 с.

6. Кто такие дата-инженеры, и как ими становятся? 2019. URL: https://habr.com/ru/company /otus/blog/452670/ (дата обращения: 16.02.20)

7. Кто такой дата-сайентист. 2018. URL: https://thecode.media/data-science/ (дата обращения: 16.02.20)

8. Новый маркетинг - Newmarketing, 2012, 2: 1. Электронный журнал. URL: https://newmarketing.ru/news/2012/09/19/462/ (дата обращения: 16.02.2020)

9. Паклин Н., Орешков В. 2013. Бизнес-аналитика. От данных к знаниям. СПб., Питер, 704 с.

10. Рагимова С. Глоссарий цифровой экономики. URL: https://www.forbes.ru/brandvoice/ sap/356277-glossariy-cifrovoy-ekonomik (дата обращения: 16.02.2020)

11. Румянцев В., Биленко П., Галюжин А. 2018. Выступления на круглом столе «Глоссарий цифровой экономики». URL: https://digital.ac.gov.ru/news/621/ (дата обращения: 16.02.2020)

12. Специалист по изучению данных (data scientist). 2019. URL: http://www.tadviser.ru /a/147974 (дата обращения: 16.02.2020)

13. ТАКСИМО. Официальный сайт «Lyft» такси в Москве. URL: https: //www.taximmo.ru/lyft-taksi-oficialnyj-sajt (дата обращения: 16.02.20)

14. Что такое Business Intelligence. 2015. URL: https://habr.com /en/company/navicon/blog /250875/ (дата обращения: 16.02.20)

15. Banafa A. 2016. The Internet of Everything (IoE). Available at: https://www.bbvaopenmind.com /en/technology/digital-world/the-internet-of-everything-ioe/ (accessed: 16.02.2020).

16. Business inteligence. 2019. Academic Dictionaries and Encyclopedias. Available at: https://translate.academic.ru/business\%20intelligence/en/ru/(accessed: 16.02.2020).

17. Business Intelligence vs Data Science - 4 Ways to Tell Them Apart. 2019. Available at: https://data-flair.training/blogs/business-intelligence-vs-data-science (accessed: 16.02.2020).

18. Chen J. 2020. Internet of Energy (IoE). Available at: https://www.investopedia.com /terms/i/internet-energy-ioe.asp(accessed: 16.02.2020).

19. Clark J. 2016. What is the Internet of Things? Available at: https://www.ibm.com/blogs/internet-of-things/what-is-the-iot/ (accessed: 16.02.2020).

20. GriffithE. 2016. WhatisCloudComputing? Available at: https://www.pcmag.com /news/what-is-cloud-computing (accessed: 16.02.2020).

21. In-memoryprocessing. Wikipedia. Available at: https://en.wikipedia.org/wiki/Inmemory_processing (accessed: 16.02.2020). 
22. Internetofthings. 2018. Medium. Internet Journal. Available at: https://medium.com /@LNMHacks/internet-of-things-59774507808a (accessed :16.02.2020).

23. Internetofthings (IoT). 2020. Available at: https://internetofthingsagenda.techtarget.com /definition/Internet-of-Things-IoT(accessed: 16.02.2020).

24. Kenton W. 2020. The Internet of Things (IoT). Available at: https://www.investopedia.com /terms/i/internet-things.asp(accessed: 16.02.2020).

25. Lift Delivery. 2019.Does it exist? Available at: https://www.ridester.com/lyft-delivery/ (accessed: 16.02.2020).

26. Melenciuc D. 2005. A Reader in English Lexicology. Moldova State University, Available at: https://ru.scribd.com/document/45834917/Lexicology-2005 (accessed: 16.02. 2020).

27. Mozyrko B. 2015. Putting Big Data in Context. Available at: https://www.uxbooth.com/articles/putting-big-data-in-context/(accessed: 16.02.2020).

28. RuizJ. What is SoLoMo and Why is it Important to Marketers? Available at: https://nealschaffer.com/solomo/ (accessed: 16.02.2020).

29. Verma A. 2018. 101 Big Data Terms You Should Know. Available at: https://www.whizlabs.com/blog/big-data-terms/(accessed: 16.02.2020).

30. Whatis Data Engineering? 2018. Available at: https:/www.datacamp.com/community /blog/data-engineering (accessed: 16.02.2020).

\section{Список литературы}

1. АртемьевВ. 2003. Что такое Business Intelligence? Открытые системы. СУБД. № 04. URL: https://www.osp.ru/os/2003/04/182900 (дата обращения: 16.02.20)

2. Что такое цифровая экономика? Тренды, компетенции, измерение. 2019. Доклады к XX Апр. междунар. науч. конф. по проблемам развития экономики и общества, Москва, 9-12 апр. 2019 г. Под ред.: Л.М Гохберг. М., Изд. дом Высшей школы экономики, 82 с. Электронная книга.URL: https://www.hse.ru/data/2019/04/12/1178004671/2\%20\%D0\%A6\%D0\%B8\%D1\%84\%D1 $\% 80 \% \mathrm{D} 0 \% \mathrm{BE} \% \mathrm{D} 0 \% \mathrm{~B} 2 \% \mathrm{D} 0 \% \mathrm{~B} 0 \% \mathrm{D} 1 \% 8 \mathrm{~F} \% \mathrm{D} 1 \% 8 \mathrm{D} \% \mathrm{D} 0 \% \mathrm{BA} \% \mathrm{D} 0 \% \mathrm{BE} \% \mathrm{D} 0 \% \mathrm{BD} \% \mathrm{D} 0 \% \mathrm{BE} \% \mathrm{D} 0$ $\% \mathrm{BC} \% \mathrm{D} 0 \% \mathrm{~B} 8 \% \mathrm{D} 0 \% \mathrm{BA} \% \mathrm{D} 0 \% \mathrm{~B} 0 . p d f$ (дата обращения: 16.02.2020)

\section{References}

1. Artem'ev V. 2003.Chto takoe Business Intelligence? [What is Business Intelligence]. Available at: https://www.osp.ru/os/2003/04/182900 (accessed: 16.02.2020).

2. What is the digital economy? Trends, competencies, and measurement. 2019. Reports by XX, APR. Intern. scientific conference on problems of economic and social development, Moscow, April 912, 2019 / G.I. Abdrahmanova, K. O. Vishnevskij, L. M. Gohberg et al. Ed. L.M Gohberg. M., Publ. Vysshej shkoly jekonomiki, 82 p. E-book. Available at: https://www.hse.ru /data/2019/04/12/1178004671/2\%20\%D0\%A6\%D0\%B8\%D1\%84\%D1 \%80\%D0\%BE\%D0\%B2\%D0 \%B0\%D1\%8F_\%D1\%8D\%D0\%BA\%D0\%BE\%D0\%BD\%D0\%BE\%D0\%BC\%D0\%B8\%D0\%BA\%D0 \%B0.pdf(accessed: 16.02 .2020$)$

\section{ИНФОРМАЦИЯ ОБ АВТОРАХ}

Глушкова Надежда Маматкуловна, кандидат филологических наук, старший преподаватель Департамента языковой подготовки Финансового университета при Правительстве Российской Федерации, г. Москва, Россия

Харченко Марина Григорьевна, старший преподаватель Департамента языковой подготовки Финансового университета при Правительстве Российской Федерации, г. Москва, Россия

\section{INFORMATION ABOUT THE AUTHORS}

Nadezhda M. Glushkova, candidate of Philological Sciences, senior lecturer at the Department of language training, Financial University under the Government of the Russian Federation, Moscow, Russia

Marina G. Kharchenko, senior lecturer at the Department of language training, Financial University under the Government of the Russian Federation, Moscow, Russia 